\title{
SASS6 overexpression is associated with mitotic chromosomal abnormalities and a poor prognosis in patients with colorectal cancer
}

\author{
KAZUYA SHINMURA ${ }^{1}$, HISAMI KATO ${ }^{1}$, YUICHI KAWANISHI ${ }^{2}$, KIYOKO NAGURA ${ }^{1}$, TAKAHARU KAMO ${ }^{1}$, \\ YUSUKE OKUBO $^{1}$, YUSUKE INOUE ${ }^{1}$, NOBUYA KURABE ${ }^{1}$, CHUNPING DU $^{1}$, MORIYA IWAIZUMI ${ }^{1}$, \\ KIYOTAKA KURACHI $^{3}$, TOSHIO NAKAMURA ${ }^{4}$ and HARUHIKO SUGIMURA ${ }^{1}$
}

${ }^{1}$ Department of Tumor Pathology, ${ }^{2}$ Research Equipment Center, and ${ }^{3}$ Department of Surgery 2, Hamamatsu University School of Medicine, Hamamatsu; ${ }^{4}$ Department of Surgery, Fujieda Municipal General Hospital, Fujieda, Shizuoka, Japan

Received March 4, 2015; Accepted April 28, 2015

DOI: $10.3892 /$ or.2015.4014

\begin{abstract}
Spindle assembly abnormal protein 6 homolog (SASS6) plays an important role in the regulation of centriole duplication. To date, the genetic alteration of SASS6 has not been reported in human cancers. In the present study, we examined whether SASS6 expression is abnormally regulated in colorectal cancers (CRCs). Increased SASS6 mRNA and protein expression levels were observed in $49(60.5 \%)$ of the 81 primary CRCs and $11(57.9 \%)$ of the 19 primary CRCs, respectively. Moreover, the upregulation of SASS6 mRNA expression was statistically significant $(\mathrm{P}=0.0410)$. Next, using DLD-1 colon cancer cells inducibly expressing SASS6, SASS6 overexpression was shown to induce centrosome amplification, mitotic abnormalities such as chromosomal misalignment and lagging chromosome, and chromosomal numerical changes. Furthermore, SASS6 overexpression was associated with anaphase bridge formation, a type of mitotic structural abnormality, in primary CRCs $(\mathrm{P}<0.01)$. SASS6 upregulation in colon cancer was also revealed in the Cancer Genome Atlas (TCGA) data and was shown to be an independent predictor of poor survival (multivariate analysis: hazard ratio, 2.805; 95\% confidence interval, 1.244-7.512; $\mathrm{P}=0.0112$ ). Finally, further analysis of the TCGA data demonstrated SASS6 upregulation in a modest manner in 8 of 11 cancer types other than colon cancer, and SASS6 upregulation was found to be associated with a poor survival outcome in patients with kidney renal cell carcinoma and lung adenocarcinoma. Our present findings revealed that the upregulation of SASS6 expression is involved in the pathogenesis of CRC and is associated with a poor prognosis among patients with colon cancer. They also suggest
\end{abstract}

Correspondence to: Dr Kazuya Shinmura, Department of Tumor Pathology, Hamamatsu University School of Medicine, Hamamatsu, Shizuoka 431-3192, Japan

E-mail: kzshinmu@hama-med.ac.jp

Key words: SASS6, centrosome amplification, colorectal cancer, overexpression, prognosis that SASS6 upregulation is a genetic abnormality relatively common in human cancer.

\section{Introduction}

Chromosome instability (CIN) is characterized by chromosomal numerical and/or structural changes and can be caused by chromosomal missegregation during mitosis $(1,2)$. CIN is observed in diverse human cancers and is associated with a poor patient prognosis in some types of cancer (3-5). Centrosome amplification is one factor that contributes to CIN through chromosomal missegregation in cancerous cells (6-8). The centrosome, a major microtubule-organizing center, is composed of a pair of centrioles and surrounding protein aggregates, and each cell is maintained so that it has one or two centrosomes during the cell cycle $(7,9)$. When centrosome amplification ( $\geq 3$ centrosomes in a cell) occurs, it causes an increase in aberrant mitotic spindle formation, merotelic kinetochore-microtubule attachment errors and lagging chromosome formation. Such mitotic abnormalities can cause chromosome segregation errors leading to CIN (6-10).

Spindle assembly abnormal protein 6 homolog (SASS6) is a centrosomal protein that, together with PLK4, CEP135, CENPJ (also known as CPAP) and STIL, is involved in regulating the number of centrosomes in human cells (11-14). The forced overexpression of SASS6 leads to centrosome amplification $(11,12,15,16)$. Notably, the abnormal expression of PLK4, resulting in the induction of centrosome amplification, has been reported in several types of cancers $(17,18)$. Moreover, genetic abnormalities of various types in other genes that play roles in centrosome regulation, such as AURKA amplification and SKA1 overexpression, have also been reported in human cancers $(19,20)$. At present, however, abnormalities in SASS6 expression or SASS6 somatic mutations have not been reported for any human cancers. Since CIN is frequently observed in colorectal cancer (CRC) $(3,5)$, we hypothesized that SASS6 abnormalities may be involved in the induction of CIN in CRC. Therefore, in the present study, we first examined whether SASS6 is aberrantly expressed in human primary CRCs. Since the upregulation of SASS6 
expression was detected in CRCs, we next showed that SASS6 overexpression induced centrosome amplification, mitotic abnormalities, and CIN in colon cancer cells. SASS6 upregulation in colon cancer was also revealed using data from the Cancer Genome Atlas (TCGA) database and was found to be associated with a relatively poor survival outcome in analyses using both the TCGA and Gene Expression Omnibus (GEO) data. Finally, SASS6 expression was shown to be upregulated in a modest manner not only in colon cancer, but also in 8 other distinct cancer types, although the incidence of somatic SASS6 mutations was exremely low.

\section{Materials and methods}

Tissue samples. CRC tissues and corresponding normal colorectal tissues were obtained from primary CRC patients treated at Hamamatsu University Hospital (Japan) for use in a quantitative reverse-transcription-polymerase chain reaction (qRT-PCR) analysis $(\mathrm{n}=81)$ and western blot analysis $(\mathrm{n}=19)$. The present study design was approved by the Institutional Review Board of the Hamamatsu University School of Medicine.

$q R T-P C R$. Total RNA was extracted using an RNeasy kit (Qiagen, Valencia, CA, USA) or an Isogen kit (Nippon Gene, Tokyo, Japan) and converted to cDNA using the SuperScript First-Strand Synthesis system for RT-PCR (Invitrogen, Carlsbad, CA, USA). The expression levels of the SASS6 and GAPDH mRNA transcripts were measured using real-time qRT-PCR with the cDNA, a set of primers, the QuantiTect SYBR-Green PCR kit (Qiagen), and a LightCycler instrument (Roche, Palo Alto, CA, USA). The following PCR primers were used for the SASS6 transcripts: 5'-CCA GAA TAC CTT CCC TCA TTC G-3' and 5'-GTT GCT CCT GAC TGA ACA TCT CC-3'. The PCR primer sequences for the GAPDH transcripts were previously described (21). The relative amounts of SASS6 transcript were normalized to those of the GAPDH transcript. The $\mathrm{T} / \mathrm{N}$ values were calculated by dividing the normalized transcript amounts in the cancerous tissue (T) of primary cancers by the amounts in the corresponding noncancerous tissue $(\mathrm{N})$.

Western blot analysis. Tissues or cultured cells were lysed in a buffer containing $50 \mathrm{mM}$ HEPES-KOH $(\mathrm{pH} 7.5)$, $150 \mathrm{mM} \mathrm{NaCl}, 0.1 \%$ sodium dodecyl sulfate, $1 \%$ Triton X-100, $0.5 \%$ sodium deoxycholate, $100 \mathrm{mM}$ sodium fluoride, $1 \mathrm{mM}$ sodium orthovanadate and protease inhibitor cocktail (Sigma-Aldrich, St. Louis, MO, USA). Western blot analysis using an anti-SASS6 polyclonal antibody (Novus Biologicals, Littleton, CO, USA) or an anti- $\beta$-tubulin monoclonal antibody (clone 2-28-33; Sigma-Aldrich) was performed as described previously (21). Immunoreactivity was visualized using an ECL chemiluminescence system (GE Healthcare Bio-Science, Piscataway, NJ, USA). ImageJ software (National Institutes of Health, Bethesda, MD, USA) was used to measure protein expression levels.

Establishment of stable inducible cell lines. The human colon cancer cell line DLD-1 was obtained from the American Type Culture Collection (ATCC, Manassas, VA, USA). The cells were maintained at $37^{\circ} \mathrm{C}$ in RPMI-1640 medium supplemented with $10 \%$ fetal bovine serum and penicillin/streptomycin under a 5\% $\mathrm{CO}_{2}$ atmosphere. DLD-1 cells were transfected with a cumate switch inducible vector for SASS6 expression, which was constructed using a GFP-SASS6 plasmid (13) kindly provided by Prof. T.K. Tang as a PCR template, together with the piggyBac transposase vector (System Biosciences, Mountain View, CA, USA). To establish stable inducible cell lines, positively transposed cells were selected using $1 \mu \mathrm{g} / \mathrm{ml}$ puromycin (Clontech, Palo Alto, CA, USA). Since the inducible piggyBac vector features a tight cumate switch combined with the EF1-CymR repressor-T2A-Puro cassette to establish stable cell lines, the addition of cumate solution (System Biosciences) to the puromycin-selected cells led to the induction of SASS6 expression.

Indirect immunofluorescence analysis. Cells were fixed with methanol and permeabilized with $1 \%$ Nonidet P-40 solution. After blocking with normal goat serum, the cells were probed with anti- $\gamma$-tubulin monoclonal antibody (GTU88; Sigma-Aldrich). Indirect immunofluorescence labeling was performed by exposure to the Alexa Fluor 594-conjugated secondary antibody (Molecular Probes, Eugene, OR, USA), and the nuclei were stained with 4',6-diamidino-2-phenylindole (DAPI) (Sigma-Aldrich). The cells were examined under a fluorescence microscope (Olympus BX-51-FL; Olympus, Tokyo, Japan) equipped with epifluorescence filters and a photometric CCD camera (Sensicam; PCO Company, Kelheim, Germany).

Fluorescence in situ hybridization (FISH) analysis. Trypsinized cells were treated with $0.075 \mathrm{M} \mathrm{KCl}$ hypotonic solution and fixed in Carnoy's fixative. The fixed cell suspension was spread onto glass slides, and the slides were then hybridized with a Spectrum Orange-labeled probe for the centromere locus on chromosome 17 [Centromere enumeration probe 17 (CEP17); Joko, Tokyo, Japan], as previously described (22). DAPI was used for nuclear staining. The cells were examined under a fluorescence microscope as described in the previous section.

Counting of anaphase bridges in primary CRC. Formalin-fixed paraffin-embedded tissue sections were stained with hematoxylin and eosin (H\&E), and a chromatin bridge between two anaphase plates was counted as an anaphase bridge under a microscope.

Collection of publicly available gene expression and somatic mutation data. Gene expression data for 5,376 samples and somatic mutation data for 4,025 samples of 12 cancer types (TCGA public data available in April 2014) were collected from the TCGA data portal (https://tcga-data.nci.nih.gov/tcga/). The patients' clinical data were also collected. The expression data were obtained as processed RNA-sequence (RNA-seq) data in the form of RNA-seq by Expectation Maximization (RSEM), which is based on a generative probabilistic model of maximum expectation (23). The somatic mutation data were obtained in the form of a mutation annotation format (MAF) file. Cancers from the following organs were analyzed: urinary bladder (BLCA), breast (BRCA), head and neck (HNSC), kidney 

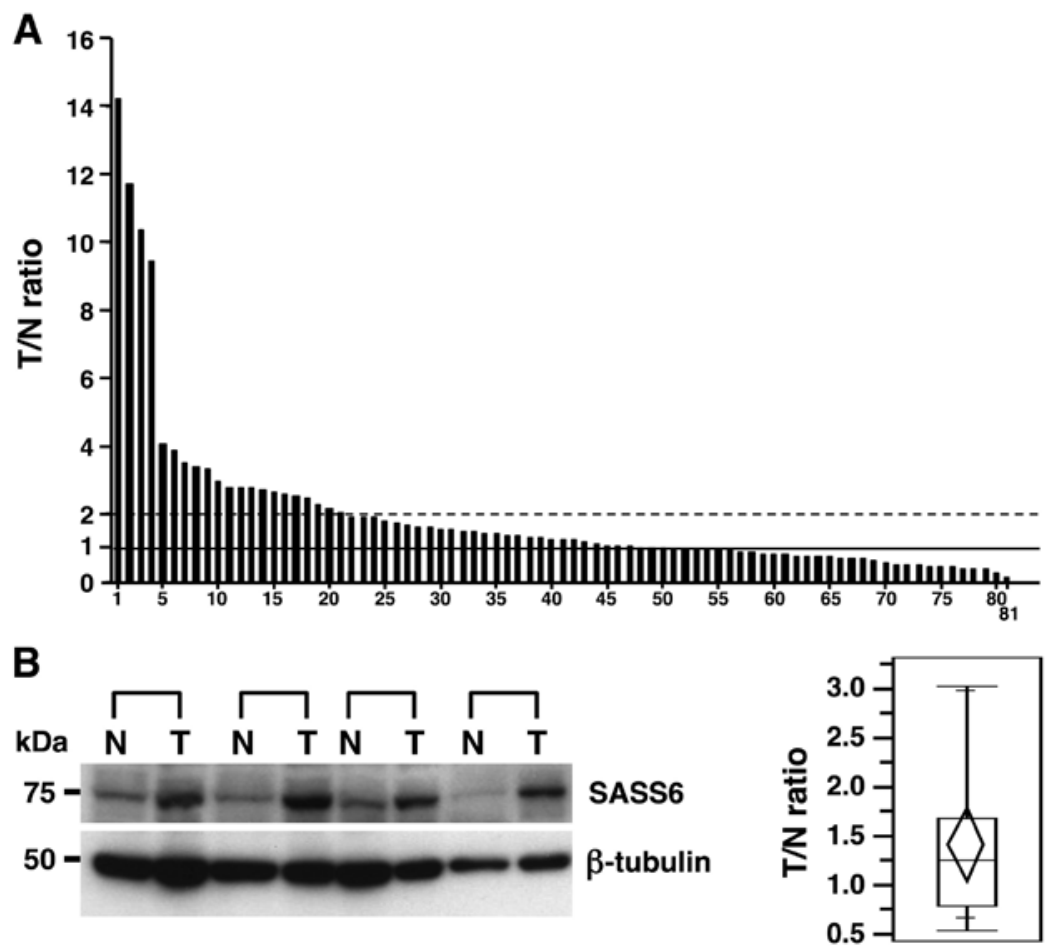

Figure 1. Upregulation of SASS6 expression level in primary CRC. (A) Upregulation of the SASS6 mRNA expression level in primary CRC. The mRNA expression level of SASS6 was compared in cancerous tissues from 81 primary CRCs and corresponding non-cancerous colorectal tissues using a real-time qRT-PCR analysis. After normalizing the amounts of SASS6 transcripts to the amounts of GAPDH transcripts, the T/N values were calculated by dividing the amount of normalized transcripts in the cancerous tissue by the amount in the corresponding non-cancerous tissue. The differences between the normalized SASS6 mRNA levels in the cancerous tissues and the corresponding non-cancerous tissues were then statistically analyzed using the Wilcoxon matched pairs test $(\mathrm{P}=0.0410)$. (B) Upregulation of SASS6 protein expression level in primary CRC. Representative results of the western blot analysis using anti-SASS6 antibody for the examination of the SASS6 protein expression level (left panel). After normalizing the amount of SASS6 protein to the amount of $\beta$-tubulin, the $\mathrm{T} / \mathrm{N}$ values were calculated by dividing the amount of normalized protein in the cancerous tissue by the amount in the corresponding non-cancerous tissue. The $\mathrm{T} / \mathrm{N}$ values are shown as a box-chart in the right panel (average, 1.42).

(KICH, KIRC and KIRP), lung (LUAD, LUSC), prostate (PRAD), thyroid gland (THCA) and uterine corpus (UCEC).

Microarray-based gene expression data for 177 CRCs and the corresponding patient clinical data, which were previously published by Smith et al (24), were also downloaded from the GEO at the National Center for Biotechnology Information (NCBI).

Statistical analysis. The statistical analysis was performed using an unpaired t-test, Mann-Whitney U test, or Wilcoxon matched pairs test. Overall survival curves were constructed using the Kaplan-Meier method, and the log-rank test was used to evaluate the differences between the curves. The hazard ratio (HR) was calculated using the Cox proportional hazard model in both univariate and multivariate analyses. JMP version 9.0 software (SAS Institute, Cary, NC, USA) was used for the analyses. $\mathrm{P}<0.05$ was considered statistically significant.

\section{Results}

Upregulation of SASS6 $m R N A$ and protein expression in a subset of primary CRCs. To investigate the status of SASS6 expression in human primary CRCs, we examined the expression of SASS6 mRNA in 81 primary CRCs using a real-time qRT-PCR analysis and calculated the ratio of the level of SASS6 mRNA expression in the cancerous tissues to that in the corresponding non-cancerous tissues ( $\mathrm{T} / \mathrm{N}$ ratio).
An increased SASS6 expression level (T/N value $>1)$ was observed in $49(60.5 \%)$ of the 81 primary CRCs (Fig. 1A). In particular, $21(25.9 \%)$ of the 81 cases had a $\mathrm{T} / \mathrm{N}$ value $\geq 2$ for the SASS6 expression level. Moreover, a significant difference in the SASS6 expression level was detected between the cancerous tissues and the corresponding non-cancerous tissues when examined using statistical analysis $(\mathrm{P}=0.0410$, Wilcoxon matched pairs test). These results suggested that the expression of SASS6 mRNA transcripts is upregulated in primary CRC. We next examined the expression of SASS6 protein in 19 primary CRCs using a western blot analysis with the anti-SASS6 antibody and calculated the ratio of the level of SASS6 protein expression in the cancerous tissues to the level in the corresponding non-cancerous tissues ( $\mathrm{T} / \mathrm{N}$ ratio). A semiquantitative analysis showed an increased SASS6 protein expression level $(\mathrm{T} / \mathrm{N}$ value $>1)$ in $11(57.9 \%)$ of the 19 primary CRCs, with an average T/N value of 1.42 (Fig. 1B). Both the mRNA and protein results described above indicate that SASS6 expression was upregulated in a subset of primary CRCs.

Induction of centrosome amplification, mitotic abnormalities, and chromosomal numerical changes by SASS6 overexpression in colonic cells. To investigate the effect of SASS6 overexpression in colonic cells, we used the piggyBac transposon vector system (25) to establish human colonic cells capable of inducibly expressing SASS6. First, DLD-1 
A
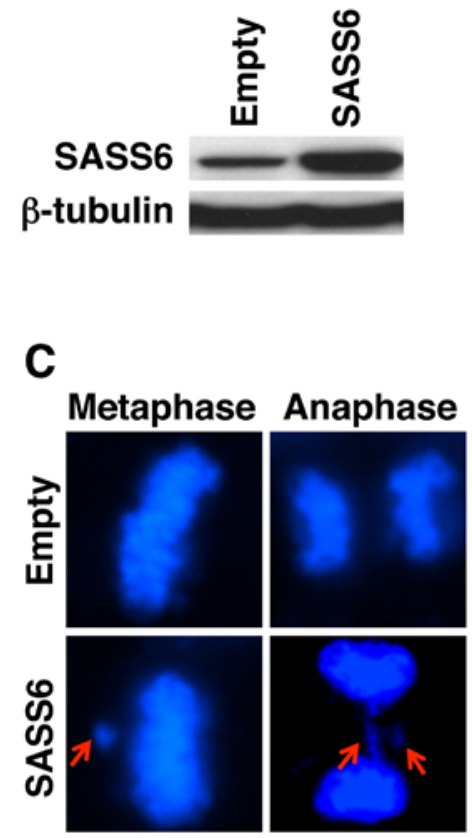

B
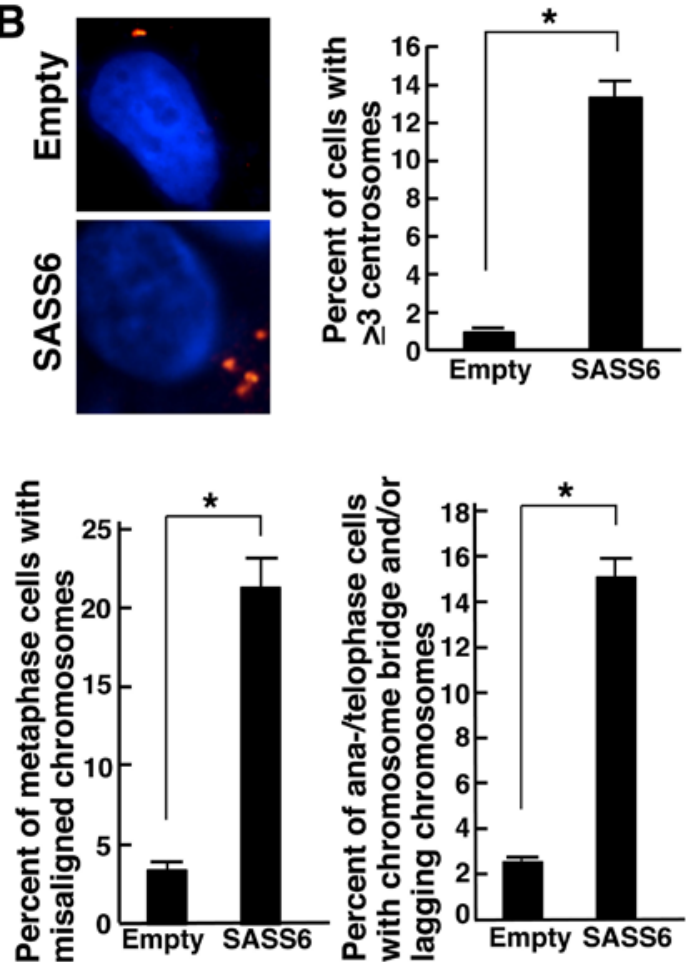
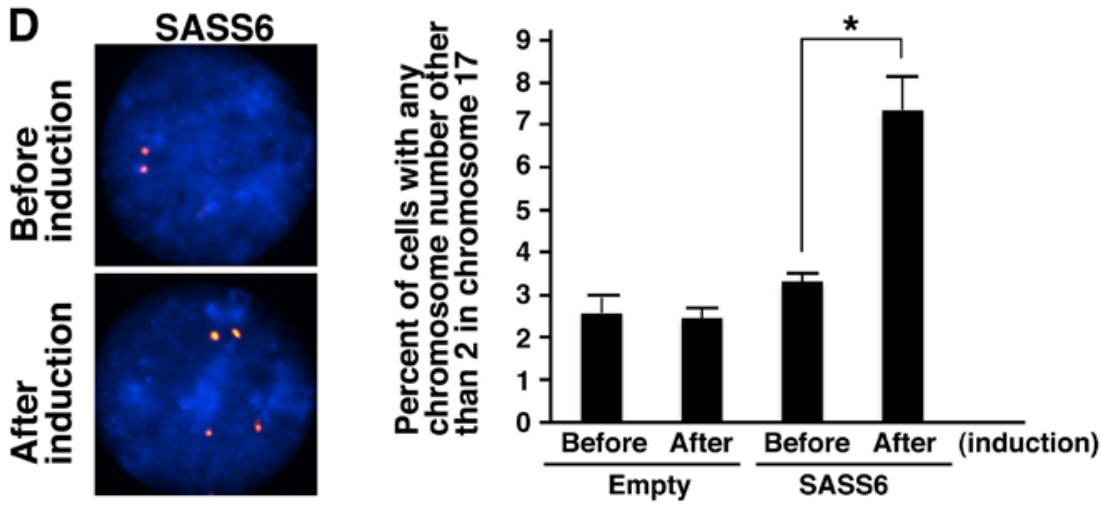

Figure 2. Induction of centrosome amplification, mitotic chromosome abnormalities and chromosomal numerical changes in response to SASS6 overexpression in colon cancer cells. (A) Detection of SASS6 proteins in cumate-inducible stable DLD-1 colon cancer cell lines designed to express SASS6 in the presence of cumate; the SASS6 proteins were detected using western blot analysis with an anti-SASS6 antibody. Lysates from empty vector-transposed cells and cells inducibly expressing SASS6 were analyzed. $\beta$-tubulin protein was also analyzed as an internal control. (B) Induction of centrosome amplification in DLD-1 cells as a result of SASS6 overexpression. At $72 \mathrm{~h}$ after cumate addition, the cells were immunostained with mouse anti- $\gamma$-tubulin monoclonal antibody (red). The nuclei were stained with DAPI (blue). The number of centrosomes per cell was counted, and the counts are shown in the right bar graph. A t-test was performed for the statistical analysis $(\mathrm{P}<0.001)$. (C) Induction of mitotic chromosome abnormalities in response to SASS6 overexpression in DLD-1 cells. At $72 \mathrm{~h}$ after cumate addition, the cells were fixed and the nuclei were stained with DAPI (blue). The percentages of metaphase cells with misaligned chromosomes were determined, and the percentages are shown in the left bar graph. The percentages of anaphase/telophase cells with chromosome bridges and/or lagging chromosomes were also determined, and the percentages are shown in the right bar graph. A t-test was used to perform the statistical analysis in each comparison $(\mathrm{P}<0.001)$. (D) Induction of chromosomal numerical changes in response to SASS6 overexpression in colon cancer cells. At $72 \mathrm{~h}$ after cumate addition, the DLD-1 cells were replated in fresh medium without cumate, cultured for an additional $72 \mathrm{~h}$, and then subjected to FISH analysis using a Spectrum Orange-labeled probe for the centromere locus on chromosome 17, CEP17. Cells before cumate induction were also subjected to FISH analysis. The nuclei were stained with DAPI (blue). The percentages of cells with an abnormal chromosome 17 number were determined, and the percentages are shown in the right bar graph. A $t$-test was used to perform the statistical analysis $(\mathrm{P}<0.01)$

colon cancer cells were transfected with a piggyBac cumate switch inducible vector for the expression of SASS6 together with the piggyBac transposase vector; positively transposed cells were then selected using puromycin. We also prepared cells transfected with an empty (parental) piggyBac cumate switch inducible vector and transposase vector. The expression of SASS6 protein was confirmed in cells transposed with the SASS6 vector after cumate induction using western blot analysis with the anti-SASS6 antibody (Fig. 2A). Then, we examined the centrosome number in the SASS6-transposed cells after cumate induction using an immunofluorescence analysis of $\gamma$-tubulin, a major centrosomal protein $(26,27)$. The frequency of cells containing $\geq 3$ centrosomes was significantly higher among the SASS6-overexpressing cells than among the empty vector-transposed cells (average: 0.9 vs. $13.3 \%, \mathrm{P}<0.001$, t-test) (Fig. $2 \mathrm{~B}$ ). This result suggests 


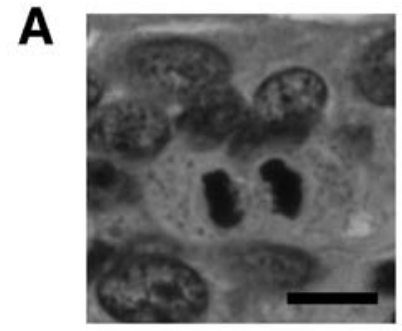

0.93

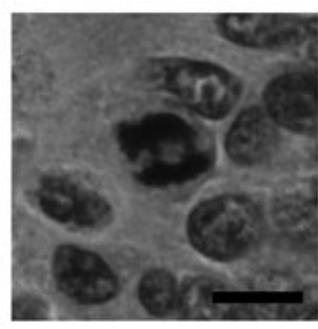

3.5

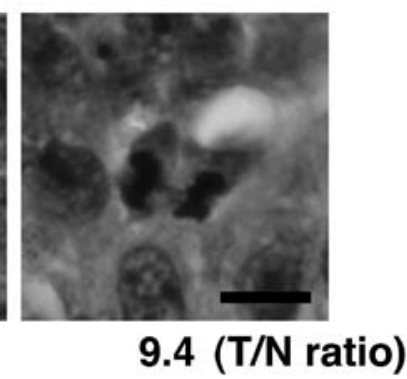

B

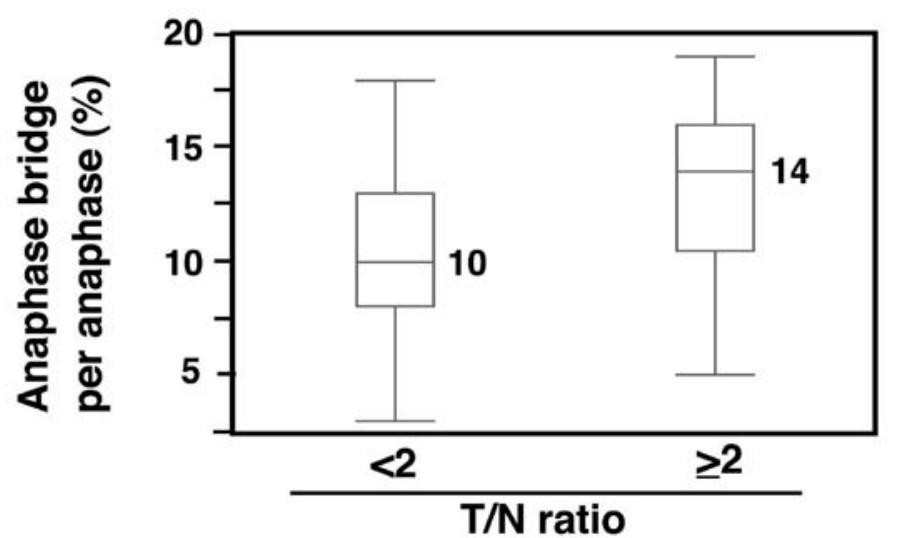

Figure 3. Increased frequency of anaphase bridges in primary CRCs with SASS6 overexpression. (A) Representative images of anaphase bridges detected in primary CRCs stained with H\&E. A normal anaphase is shown in the left panel, and anaphase bridges are shown in the middle and right panels. The T/N ratio of each CRC is provided under the panel. (B) Box-plot analysis of anaphase bridge formation in primary CRCs. A statistically significant difference in the incidence of anaphase bridge formation was detected between a lower SASS6 expression group (T/N $<2$ ) and a higher SASS6 expression group (T/N $\geq 2$ ) $(\mathrm{P}<0.01$, Mann-Whitney U test).

that the upregulation of SASS6 expression causes centrosome amplification in colonic cells. Next, we attempted to determine whether the increased frequency of centrosome amplification in the SASS6-overexpressing colonic cells resulted in mitotic chromosomal abnormalities. When mitotic phase cells were examined after cumate induction, the percentage of metaphase cells with misaligned chromosomes was significantly higher for the SASS6-overexpressing cells (average: 3.4 vs. $21.3 \%$, $\mathrm{P}<0.001$, t-test) (Fig. 2C). Moreover, the percentage of anaphase/telophase cells with a chromosome bridge and/or lagging chromosomes was also significantly higher for the SASS6-overexpressing cells (average: 2.5 vs. $15.1 \%, \mathrm{P}<0.001$, t-test) (Fig. 2C). These results suggested that SASS6 overexpression is associated with mitotic abnormalities in colonic cells. Next, to investigate the effect of SASS6 overexpression on the number of chromosomes, the chromosome 17 number per cell was compared before and after cumate induction using FISH analysis with CEP17. The results showed that the percentage of cells with an abnormal chromosome 17 number was higher for the SASS6-overexpressing cells (average: 3.3 vs. $7.5 \%, \mathrm{P}<0.01$, t-test) (Fig. 2D), suggesting that SASS6 overexpression is associated with changes in the chromosomal number (such as CIN) in colonic cells. Based on the above findings, SASS6 overexpression was thought to contribute to centrosome amplification, mitotic abnormalities and CIN in colonic cells.

Increased anaphase bridge formation in primary CRCs with SASS6 overexpression. We next investigated whether mitotic chromosomal aberrations were also associated with SASS6 overexpression in primary CRCs. The presence of bridge formation in anaphase cells was examined using H\&E-stained sections from the $81 \mathrm{CRCs}$ that were used in the SASS6 mRNA expression analysis. A T/N ratio of 2 was used as the cut-off value, such as for defining a higher SASS6 expression group $(\mathrm{T} / \mathrm{N} \geq 2)$ and a lower SASS6 expression group (T/N $<2)$. As a result, a significantly higher frequency of anaphase bridge formation was observed in the higher SASS6 expression group, compared with the lower SASS6 expression group (median: 14 vs. 10\%, P<0.01, Mann-Whitney U test) (Fig. 3). These results suggest that SASS6 overexpression is associated with anaphase bridge formation, which is a type of mitotic chromosomal aberration, in primary CRC.

Association between SASS6 overexpression and a poor survival outcome in colon cancer. Next, to obtain solid evidence of SASS6 overexpression in CRC, we examined the SASS6 expression level of another cohort of colon cancer from the TCGA dataset (28). The SASS6 expression level, as determined using an RNA-seq analysis, was significantly higher in colon adenocarcinoma $(n=260)$ than that in the non-cancerous colonic tissues $(n=41)(P<0.0001$, MannWhitney U test) (Fig. 4A). These results support our conclusion that SASS6 is overexpressed in CRC.

Next, we investigated whether the SASS6 level is associated with survival in patients with colon cancer. A Kaplan-Meier analysis for a total of 257 colon adenocarcinoma patients showed that the prognosis of patients with colon adenocarcinoma exhibiting a relatively high SASS6 expression level was significantly poorer than the prognosis of patients with colon 

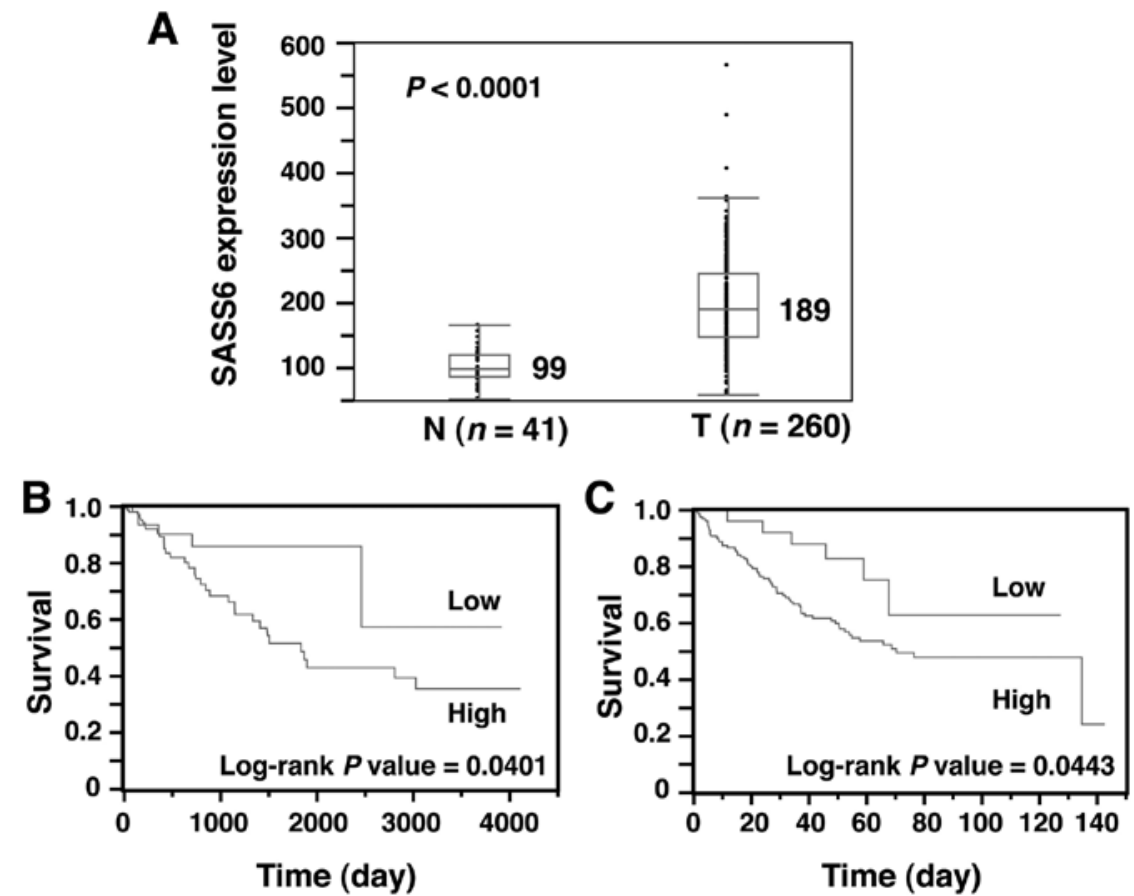

Figure 4. Association of SASS6 overexpression with a poor survival outcome in colon cancer. (A) Upregulation of SASS6 mRNA expression in colon adenocarcinoma using data from the TCGA database. A box-plot analysis showed a statistically significant difference in the level of SASS6 mRNA expression measured using RNA-seq (RSEM data) between the non-cancerous colonic tissues and colon adenocarcinomas ( $<<0.0001$, Mann-Whitney U test). (B) Impact of SASS6 overexpression on overall survival in colon adenocarcinoma patients $(n=257)$ from the TCGA database. The survival curves were generated using the Kaplan-Meier method. A cut-off value of 1.5-fold the mean SASS6 expression value in non-cancerous colonic tissue was used, and the patients were divided into a low SASS6 expression group and a high SASS6 expression group. (C) Impact of SASS6 overexpression on overall survival in colon cancer patients $(n=177)$ from the GEO database (24). The survival curves were generated using the Kaplan-Meier method. An expression level of 6.68 in the microarray-based data was used as a cut-off value, and the patients were divided into a low SASS6 expression group and a high SASS6 expression group.

Table I. Cox proportional hazard analysis of potential predictors of a poor prognosis in colon adenocarcinoma patients $(\mathrm{n}=257)$ using data from the TCGA database.

Univariate analysis Multivariate analysis

\begin{tabular}{|c|c|c|c|c|}
\hline \multirow[b]{2}{*}{ Variable } & & & \\
\hline & $\mathrm{HR}(95 \% \mathrm{CI})$ & P-value & $\mathrm{HR}(95 \% \mathrm{CI})$ & $\mathrm{P}$-value \\
\hline \multicolumn{5}{|l|}{ Gender } \\
\hline Male & $1.619(0.867-3.137)$ & 0.1317 & & \\
\hline Female & 1 & & & \\
\hline \multicolumn{5}{|c|}{ Age (years) } \\
\hline$\geq 60$ & $1.525(0.740-3.551)$ & 0.2656 & & \\
\hline$<60$ & 1 & & & \\
\hline \multicolumn{5}{|l|}{ Stage } \\
\hline III, IV & $1.999(1.054-3.914)$ & 0.0337 & $2.372(1.236-4.694)$ & 0.0093 \\
\hline $\mathrm{I}, \mathrm{II}$ & 1 & & 1 & \\
\hline \multicolumn{5}{|c|}{ SASS6 expression ${ }^{a}$} \\
\hline High & $2.415(1.090-6.396)$ & 0.0284 & $2.805(1.244-7.512)$ & 0.0112 \\
\hline Low & 1 & & 1 & \\
\hline
\end{tabular}

HR, hazard ratio; CI, confidence interval. a A cut-off value of 1.5-fold the mean SASS6 expression value in the non-cancerous colonic tissues was used.

adenocarcinoma exhibiting a relatively low SASS6 expression level ( $\mathrm{P}=0.0401$, log-rank test) (Fig. 4B). To rule out potential factors that may have confounded the SASS6 expression results, we conducted univariate and multivariate analyses for overall 
Table II. Cox proportional hazard analysis of potential predictors of a poor prognosis in colorectal cancer patients ( $\mathrm{n}=177)$ using data from the GEO database.

\begin{tabular}{|c|c|c|c|c|}
\hline \multirow[b]{2}{*}{ Variable } & \multicolumn{2}{|c|}{ Univariate analysis } & \multicolumn{2}{|c|}{ Multivariate analysis } \\
\hline & $\mathrm{HR}(95 \% \mathrm{CI})$ & P-value & HR (95\% CI) & P-value \\
\hline \multicolumn{5}{|l|}{ Gender } \\
\hline Male & $1.179(0.741-1.899)$ & 0.4901 & & \\
\hline Female & 1 & & & \\
\hline \multicolumn{5}{|c|}{ Age (years) } \\
\hline$\geq 60$ & $0.881(0.548-1.451)$ & 0.6106 & & \\
\hline$<60$ & 1 & & & \\
\hline \multicolumn{5}{|l|}{ Stage } \\
\hline III, IV & $4.220(2.454-7.733)$ & $<0.0001$ & $4.140(2.406-7.589)$ & $<0.0001$ \\
\hline I, II & 1 & & 1 & \\
\hline \multicolumn{5}{|c|}{ SASS6 expression ${ }^{\mathrm{a}}$} \\
\hline High & $2.302(1.084-5.954)$ & 0.0283 & $2.134(1.003-5.522)$ & 0.0489 \\
\hline Low & 1 & & 1 & \\
\hline
\end{tabular}

HR, hazard ratio; CI, confidence interval. aAn expression level of 6.68 was used as a cut-off value for the microarray-based data.

survival, using the Cox proportional hazard model (Table I). An advanced stage (III and IV) and a high SASS6 expression level were associated with significantly elevated risks of a poor survival outcome in both the univariate and multivariate analyses. The HR of the former and latter factors in the multivariate analyses were 2.372 [95\% confidence interval (CI), 1.2364.694, $\mathrm{P}=0.0093$ ] and 2.805 (95\% CI, 1.244-7.512, $\mathrm{P}=0.0112$ ), respectively. We also assessed the prognostic impact of SASS6 overexpression on colon cancer in another cohort collected by Smith et al (24). A Kaplan-Meier analysis of the expression and clinical data for a total of 177 colon cancer patients whose data were included in the GEO showed that SASS6 overexpression was associated with a poorer survival outcome in the patients with colon cancer ( $\mathrm{P}=0.0443$, log-rank test) (Fig. 4C). Moreover, multivariate analysis examining overall survival using the Cox proportional hazard model showed that SASS6 overexpression was associated with a significantly increased risk of a poor survival outcome (HR, 2.134; 95\% CI, 1.0035.522; $\mathrm{P}=0.0489$ ) (Table II). Thus, the results of these two cohort studies both suggest that SASS6 overexpression is an independent predictor of a poor survival outcome in patients with colon cancer.

SASS6 overexpression in diverse human cancers. Next, to investigate the SASS6 expression level in various human cancers, we examined the SASS6 expression level determined using an RNA-seq analysis across a panel of 11 distinct cancer types utilizing the TCGA data. The results showed that SASS6 expression was significantly upregulated in a modest manner in the cancerous tissues of the following 8 cancer types, compared with non-cancerous tissues (Mann-Whitney U test): bladder urothelial carcinoma $(\mathrm{P}<0.0001)$, breast invasive carcinoma $(\mathrm{P}<0.0001)$, head and neck squamous cell carcinoma $(\mathrm{P}<0.0001)$, kidney renal clear cell carcinoma $(\mathrm{P}<0.0001)$, lung adenocarcinoma $(\mathrm{P}<0.0001)$, lung squamous cell carcinoma $(\mathrm{P}<0.0001)$, prostate adenocarcinoma $(\mathrm{P}=0.0012)$, and uterine corpus endometrial carcinoma $(\mathrm{P}<0.0001)$ (Fig. 5). The result suggested that SASS6 expression is upregulated in diverse human cancers.

Association between SASS6 overexpression and a poor survival outcome in cancers other than colon cancer. We next investigated whether the SASS6 level was also associated with survival in patients with cancer other than colon cancer. A Kaplan-Meier analysis showed that SASS6 overexpression was associated with a poorer outcome in patients with kidney renal clear cell carcinoma $(\mathrm{P}=0.0011)$ (Fig. $6 \mathrm{~A})$ and those with lung adenocarcinoma ( $\mathrm{P}=0.0208)$ (Fig. 6B). Moreover, a multivariate analysis using the Cox proportional hazard model showed that SASS6 overexpression was associated with a significantly increased risk of a poor outcome in patients with kidney renal clear cell carcinoma (HR, 1.515, 95\% CI, 1.039-2.162, $\mathrm{P}=0.0316$ ) (Table III) and those with lung adenocarcinoma (HR, 1.599; 95\% CI, 1.030-2.578; $\mathrm{P}=0.0359$ ) (Table IV). These results, along with the results for colon cancer, suggest that SASS6 overexpression is an independent predictor of a poor survival outcome in patients with some types of cancers.

Low incidence of somatic SASS6 mutations in human cancer. Finally, the presence of a somatic SASS6 mutation, another type of cancer-specific genetic alteration, was examined in 12 cancer types including colon cancer using TCGA data. The incidence of somatic nonsynonymous mutations of the SASS6 gene was 0 to $3.2 \%$ among the cancers; if all the cancer cases were combined, the incidence was $0.7 \%$ ( 29 out of 4,025 analyzed cases) (Table V). These results indicate that the influence of somatic mutations on SASS6 activation or inactivation is extemely limited. 

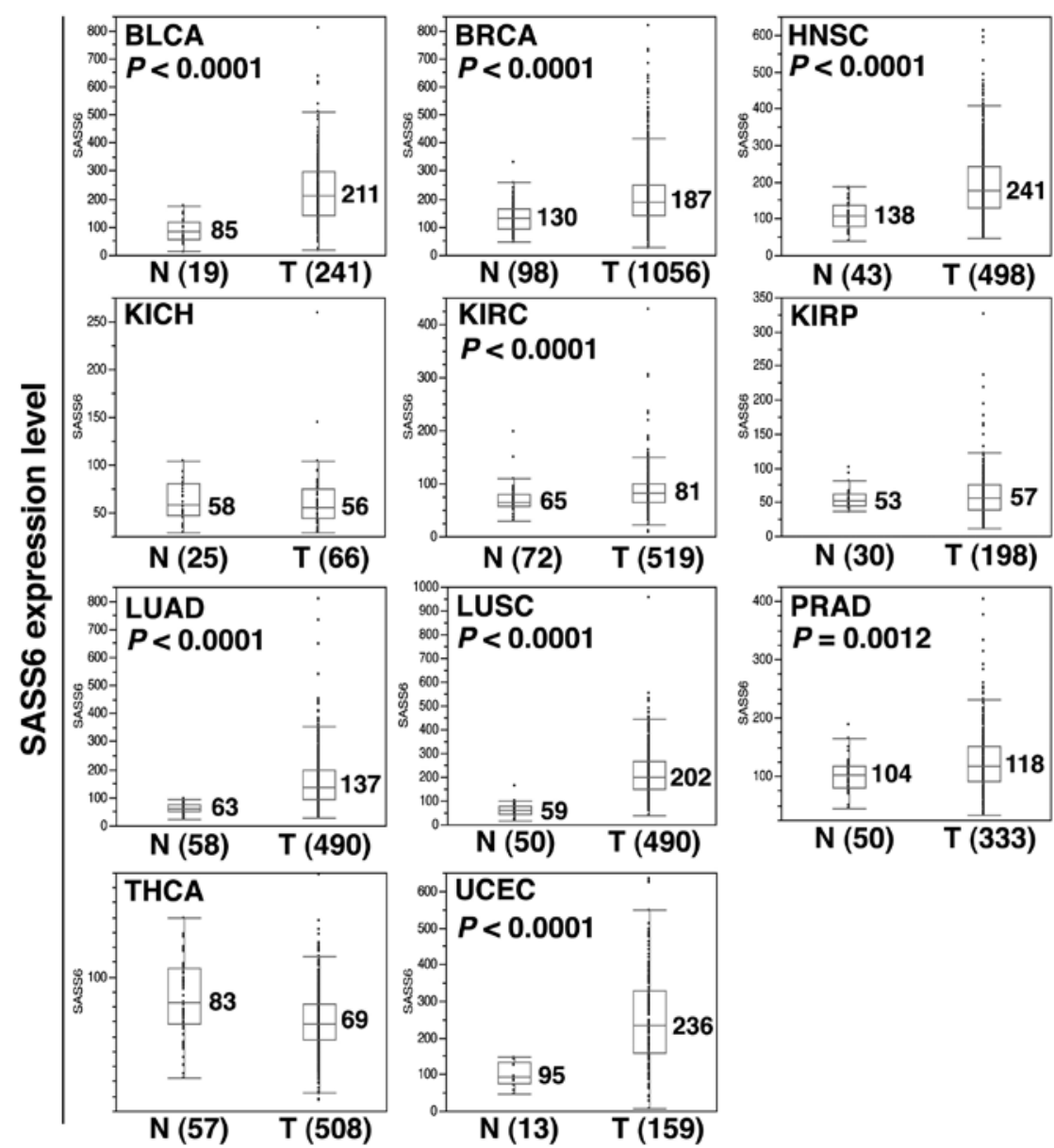

Figure 5. Upregulation of SASS6 mRNA expression in diverse human cancers. A box-plot analysis was performed for SASS6 mRNA expression data (RSEM data) from the following 11 cancer types: BLCA, bladder urothelial carcinoma; BRCA, breast invasive carcinoma; HNSC, head and neck squamous cell carcinoma; KICH, kidney chromophobe renal cell carcinoma; KIRC, kidney renal clear cell carcinoma; KIRP, kidney renal papillary cell carcinoma; LUAD, lung adenocarcinoma; LUSC, lung squamous cell carcinoma; PRAD, prostate adenocarcinoma; THCA, thyroid carcinoma; and UCEC, uterine corpus endometrial carcinoma. A Mann-Whitney U test was used to perform the statistical analysis and a P-value was provided if the upregulation of SASS6 expression was detected in the tumor.

A

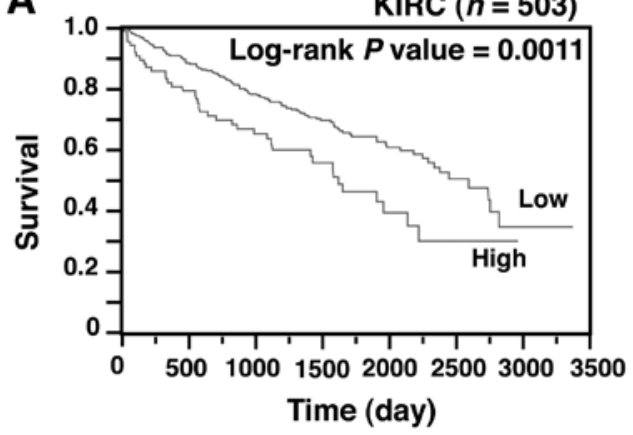

B

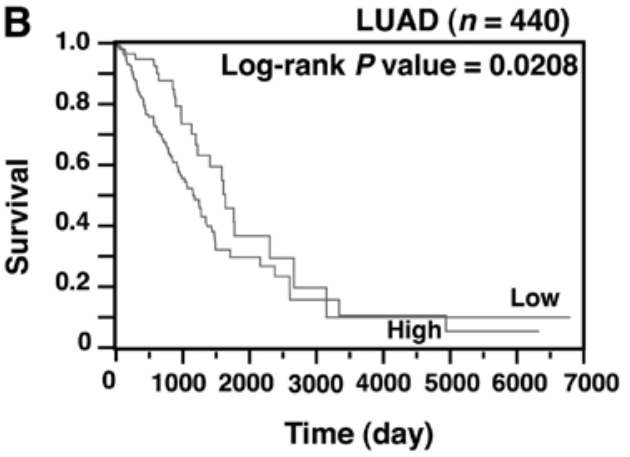

Figure 6. Association of SASS6 overexpression with a poor survival outcome in patients with kidney renal clear cell carcinoma or lung adenocarcinoma Impact of SASS6 overexpression on overall patient survival in (A) kidney renal clear cell carcinoma ( $\mathrm{n}=503)$ and $(\mathrm{B})$ lung adenocarcinoma ( $\mathrm{n}=440)$ using data from TCGA database. The survival curves were generated using the Kaplan-Meier method. A cut-off value of 1.5-fold the mean SASS6 expression value in non-cancerous kidney or lung tissue was used and the patients were divided into a low SASS6 expression group and a high SASS6 expression group.

\section{Discussion}

In the present study, the SASS6 mRNA and protein expression levels were shown to be upregulated in $\sim 60 \%$ of primary CRCs. To clarify the effect of SASS6 overexpression on colonic cells, we established DLD-1 colon cancer cells inducibly expressing SASS6; using this cell system, we then showed that SASS6 overexpression induced centrosome amplification, mitotic abnormalities such as chromosomal misalignment and lagging chromosome and CIN. In primary CRCs, moreover, 
Table III. Cox proportional hazard analysis of potential predictors of a poor prognosis in kidney renal clear cell carcinoma patients $(n=503)$ using data from the TCGA database.

\begin{tabular}{|c|c|c|c|c|}
\hline \multirow[b]{2}{*}{ Variable } & \multicolumn{2}{|c|}{ Univariate analysis } & \multicolumn{2}{|c|}{ Multivariate analysis } \\
\hline & $\operatorname{HR}(95 \% \mathrm{CI})^{\mathrm{a}}$ & P-value & HR $(95 \% \mathrm{CI})$ & P-value \\
\hline \multicolumn{5}{|l|}{ Gender } \\
\hline Male & $0.926(0.673-1.287)$ & 0.6444 & & \\
\hline Female & 1 & & & \\
\hline \multicolumn{5}{|l|}{ Years } \\
\hline$\geq 60$ & $1.800(1.294-2.538)$ & 0.0004 & $1.536(1.101-2.170)$ & 0.0112 \\
\hline$<60$ & 1 & & & \\
\hline \multicolumn{5}{|l|}{ Stage } \\
\hline III, IV & $4.194(3.012-5.919)$ & $<0.0001$ & $3.824(2.733-5.423)$ & $<0.0001$ \\
\hline $\mathrm{I}, \mathrm{II}$ & 1 & & 1 & \\
\hline \multicolumn{5}{|c|}{ SASS6 expression $^{\text {b }}$} \\
\hline High & $1.805(1.245-2.561)$ & 0.0022 & $1.515(1.039-2.162)$ & 0.0316 \\
\hline Low & 1 & & 1 & \\
\hline
\end{tabular}

${ }^{\mathrm{a}} \mathrm{HR}$, hazard ratio; CI, confidence interval. ${ }^{\mathrm{b}} \mathrm{A}$ cut-off value of 1.5-fold the mean SASS6 expression value in non-cancerous kidney tissue was used.

Table IV. Cox proportional hazard analysis of potential predictors of a poor prognosis in lung adenocarcinoma patients $(\mathrm{n}=440)$ using data from the TCGA database.

\begin{tabular}{|c|c|c|c|c|}
\hline \multirow[b]{2}{*}{ Variable } & \multicolumn{2}{|c|}{ Univariate analysis } & \multicolumn{2}{|c|}{ Multivariate analysis } \\
\hline & $\operatorname{HR}(95 \% \mathrm{CI})^{\mathrm{a}}$ & P-value & HR $(95 \% \mathrm{CI})$ & P-value \\
\hline \multicolumn{5}{|l|}{ Gender } \\
\hline Male & $0.954(0.652-1.387)$ & 0.8040 & & \\
\hline Female & 1 & & & \\
\hline \multicolumn{5}{|l|}{ Years } \\
\hline$\geq 60$ & $0.989(0.655-1.533)$ & 0.9596 & & \\
\hline$<60$ & 1 & & & \\
\hline \multicolumn{5}{|l|}{ Stage } \\
\hline III, IV & $2.712(1.835-3.974)$ & $<0.0001$ & $2.640(1.783-3.873)$ & $<0.0001$ \\
\hline $\mathrm{I}, \mathrm{II}$ & 1 & & 1 & \\
\hline \multicolumn{5}{|c|}{ SASS6 expression ${ }^{\mathrm{a}}$} \\
\hline High & $1.698(1.097-2.732)$ & 0.0167 & $1.599(1.030-2.578)$ & 0.0359 \\
\hline Low & 1 & & 1 & \\
\hline
\end{tabular}

$\mathrm{HR}$, hazard ratio; CI, confidence interval. ${ }^{a} \mathrm{~A}$ cut-off value of 1.5 -fold the mean SASS6 expression value in non-cancerous lung tissue was used.

SASS6 overexpression was shown to be associated with an increase in anaphase bridge formation. SASS6 upregulation in colon cancer was also revealed using TCGA data and was associated with a relatively poor survival outcome in analyses using both the TCGA and GEO data. Finally, SASS6 expression was shown to be upregulated in a modest manner not only in colon cancer, but also in 8 other distinct cancer types and SASS6 upregulation was also associated with a relatively poor survival outcome in patients with two cancer types other than colon cancer. These results suggest that SASS6 overexpression may be involved in the pathogenesis of CRC through the induction of centrosome amplification, mitotic abnormalities and CIN and SASS6 overexpression may be a predictor of a poor survival outcome in patients with colon cancer. The TCGA data also suggested that SASS6 overexpression is a phenomenon that is relatively common in human cancer. These findings may partly explain the earlier observation of a high prevalence of centrosome amplification and CIN in human 
Table V. Incidence of somatic nonsynonymous mutations of the SASS6 gene in human cancers using data from the TCGA database.

\begin{tabular}{|c|c|c|c|c|}
\hline Cancer type & $\begin{array}{l}\text { TCGA } \\
\text { ID }\end{array}$ & $\begin{array}{l}\text { No. of cases } \\
\text { analyzed }\end{array}$ & $\begin{array}{c}\text { No. of cases } \\
\text { with nonsynonymous } \\
\text { mutations }\end{array}$ & $\begin{array}{c}\text { Incidence } \\
(\%)\end{array}$ \\
\hline Bladder urothelial carcinoma & BLCA & 130 & 0 & 0.0 \\
\hline Breast invasive carcinoma & BRCA & 991 & 4 & 0.4 \\
\hline Colon adenocarcinoma & COAD & 269 & 7 & 2.6 \\
\hline Head and neck squamous cell carcinoma & HNSC & 509 & 1 & 0.2 \\
\hline Kidney chromophobe renal cell carcinoma & $\mathrm{KICH}$ & 66 & 0 & 0.0 \\
\hline Kidney renal clear cell carcinoma & KIRC & 235 & 1 & 0.4 \\
\hline Kidney renal papillary cell carcinoma & KIRP & 171 & 1 & 0.6 \\
\hline Lung adenocarcinoma & LUAD & 561 & 5 & 0.9 \\
\hline Lung squamous cell carcinoma & LUSC & 178 & 2 & 1.1 \\
\hline Prostate adenocarcinoma & PRAD & 261 & 0 & 0.0 \\
\hline Thyroid carcinoma & THCA & 406 & 0 & 0.0 \\
\hline Uterine corpus endometrial carcinoma & UCEC & 248 & 8 & 3.2 \\
\hline Total & & 4,025 & 29 & 0.7 \\
\hline
\end{tabular}

cancers. According to our knowledge, the present study is the first study to describe aberrant SASS6 expression in human cancer.

In the present study, SASS6 overexpression was observed in primary CRCs from both our patient series and the TCGA dataset. As demonstrated in our analysis, centrosome amplification, mitotic chromosome abnormalities, and CIN were induced by SASS6 overexpression in colonic cells, and the frequent appearance of centrosome and chromosome abnormalities in CRC has been previously reported $(3,5,29,30)$. Thus, SASS6 overexpression may contribute to the development of CRC via the induction of centrosome and chromosome abnormalities. Notably, SASS6 overexpression was also observed in 8 other cancer types including cancers of the urinary bladder, breast, uterus, head and neck, kidney, liver, lung and prostate, indicating that SASS6 upregulation is a relatively common genetic abnormality in human cancer. At present, the mechanism underlying the upregulation of SASS6 expression remains uncertain. However, some possibilities, such as the cancer-specific abnormal expression of miRNAs controlling the level of SASS6 mRNA transcripts or transcription factors regulating SASS6 expression or cancer-specific SASS6 gene amplification, can be suggested in view of previous studies regarding gene overexpression in cancer (31-33). Future studies examining these points should help to clarify the cause of SASS6 upregulation.

Our demonstration of the induction of centrosome amplification by SASS6 overexpression in colonic cells was consistent with the results of previous studies describing the induction of centrosome amplification by the ectopic expression of SASS6 in U2OS osteosarcoma cells $(11,12,15,16)$. As possible mechanisms linking centrosome amplification to CIN, centrosome amplification has been suggested to result in abnormal mitotic spindle formation and merotelic kinetochore-microtubule attachment errors leading to the formation of lagging chro- mosomes and these mitotic abnormalities evoke chromosome missegregation, such as the occurrence of chromosomal numerical changes $(6-10,34,35)$. Thus, centrosome amplification as a result of SASS6 overexpression was believed to have induced CIN in the colonic cells in the present study. In our analysis of the incidence of anaphase bridges in primary $\mathrm{CRC}$, a higher incidence of anaphase bridges was associated with SASS6 overexpression. To date, several causative events leading to anaphase bridge formation are known, including centrosome amplification as a result of the expression of oncogenic MET, the expression of human papilloma virus-16 E6 and E7 oncoprotein, the mutation of genes involved in the function of the BAF complex, replication stress, and chromosome breakage (36-40). Thus, centrosome amplification as a result of SASS6 overexpression may be related to anaphase bridge formation in primary CRC. However, we cannot completely deny the possibility that some unknown event that co-occurs with SASS6 overexpression may be responsible for anaphase bridge formation.

In the present study, SASS6 overexpression was clearly shown to be an independent predictor of a poor survival outcome among patients with cancer of the colon, kidney, and lung. In all the analyses, the disease stage was identified as an independent predictor of a poor survival outcome, suggesting that the presently performed analyses were valid. Since previous studies have shown that CIN is associated with a poor patient prognosis $(3,5)$ and an association between SASS6 overexpression and CIN was shown in the present study, CIN may be involved in the relationship between SASS6 overexpression and the poor prognosis of patients with the above-mentioned cancers. Additionally, since centrosome amplification has recently been shown to be capable of mimicking and strengthening the effects of oncogenes in triggering cellular invasion (41), this phenotype arising from centrosome amplification induced by SASS6 overexpression 
may also be involved in the relationship between SASS6 overexpression and a poor prognosis.

In contrast to SASS6 overexpression, the incidence of somatic SASS6 nonsynonymous mutations was extremely low across the 12 human cancers that were examined in this study. An activating $B R A F$ p.V600E mutation is known to be common in cancer and to cause centrosome amplification and CIN (42) and an inactivating BUB1B mutation noted in premature chromatid separation (PCS) syndrome is also known to cause centrosome amplification (43). Nevertheless, the low incidence of SASS6 mutations observed in our investigation suggests that a similar activating or inactivating mutation in the SASS6 gene is either rare or non-existent in human cancer. In conjunction with the SASS6 expression data, the above findings suggest that SASS6 overexpression, but not SASS6 mutation, is likely to be frequently involved in the pathogenesis of human cancer.

In conclusion, we demonstrated that SASS6 expression is upregulated in primary $\mathrm{CRC}$ and that SASS6 overexpression induces centrosome amplification, mitotic abnormalities, and $\mathrm{CIN}$ in colonic cells and is thus a predictor of a poor survival outcome among patients with colon cancer. We also demonstrated that SASS6 overexpression is common in diverse human cancers and is associated with a poor prognosis in two more cancers. These findings suggest that SASS6 overexpression may be involved in the pathogenesis of diverse human cancers, particularly CRC.

\section{Acknowledgements}

We would like to thank Professor T.K. Tang (Institute of Biomedical Sciences, Academia Sinica, Taiwan, ROC) for providing us with the GFP-SASS6 vector. The present study was supported in part by a Grant-in-Aid from the Ministry of Health, Labour and Welfare (21-1, 10103838), the Japan Society for the Promotion of Science (25460476), the Ministry of Education, Culture, Sports, Science and Technology (221S0001), the Takeda Science Foundation and the Smoking Research Foundation.

\section{References}

1. Thompson SL, Bakhoum SF and Compton DA: Mechanisms of chromosomal instability. Curr Biol 20: R285-R295, 2010.

2. Pfau SJ and Amon A: Chromosomal instability and aneuploidy in cancer: From yeast to man. EMBO Rep 13: 515-527, 2012.

3. Watanabe T, Kobunai T, Yamamoto Y, Matsuda K, Ishihara S, Nozawa K, Yamada H, Hayama T, Inoue E, Tamura J, et al: Chromosomal instability (CIN) phenotype, CIN high or CIN low, predicts survival for colorectal cancer. J Clin Oncol 30 2256-2264, 2012.

4. Vincent-Salomon A, Benhamo V, Gravier E, Rigaill G, Gruel N, Robin S, de Rycke Y, Mariani O, Pierron G, Gentien D, et al: Genomic instability: A stronger prognostic marker than proliferation for early stage luminal breast carcinomas. PLoS One 8: e76496, 2013.

5. Hveem TS, Merok MA, Pretorius ME, Novelli M, Bævre MS, Sjo OH, Clinch N, Liest $\varnothing 1 \mathrm{~K}$, Svindland A, Lothe RA, et al Prognostic impact of genomic instability in colorectal cancer. Br J Cancer 110: 2159-2164, 2014

6. Ganem NJ, Godinho SA and Pellman D: A mechanism linking extra centrosomes to chromosomal instability. Nature 460 278-282, 2009

7. Fukasawa K: Oncogenes and tumour suppressors take on centrosomes. Nat Rev Cancer 7: 911-924, 2007.

8. Krämer A, Maier B and Bartek J: Centrosome clustering and chromosomal (in)stability: A matter of life and death. Mol Oncol 5: 324-335, 2011.
9. Nigg EA and Raff JW: Centrioles, centrosomes, and cilia in health and disease. Cell 139: 663-678, 2009.

10. Shinmura K and Sugimura H: Centrosome abnormality and human lung cancer. In: Lung Diseases - Selected State of the Art Reviews. Irusen EM (ed). InTech, Rijeka, pp171-188, 2012.

11. Leidel S, Delattre M, Cerutti L, Baumer K and Gönczy P: SAS-6 defines a protein family required for centrosome duplication in C. elegans and in human cells. Nat Cell Biol 7: 115-125, 2005.

12. Arquint C, Sonnen KF, Stierhof YD and Nigg EA: Cell-cycleregulated expression of STIL controls centriole number in human cells. J Cell Sci 125: 1342-1352, 2012.

13. Lin YC, Chang CW, Hsu WB, Tang CJ, Lin YN, Chou EJ, Wu CT and Tang TK: Human microcephaly protein CEP135 binds to hSAS-6 and CPAP, and is required for centriole assembly. EMBO J 32: 1141-1154, 2013

14. Keller D, Orpinell M, Olivier N, Wachsmuth M, Mahen R, Wyss R, Hachet V, Ellenberg J, Manley S and Gönczy P: Mechanisms of HsSAS-6 assembly promoting centriole formation in human cells. J Cell Biol 204: 697-712, 2014.

15. Strnad P, Leidel S, Vinogradova T, Euteneuer U, Khodjakov A and Gönczy P: Regulated HsSAS-6 levels ensure formation of a single procentriole per centriole during the centrosome duplication cycle. Dev Cell 13: 203-213, 2007.

16. Comartin D, Gupta GD, Fussner E, Coyaud É, Hasegan M, Archinti M, Cheung SW, Pinchev D, Lawo S, Raught B, et al: CEP120 and SPICE1 cooperate with CPAP in centriole elongation. Curr Biol 23: 1360-1366, 2013.

17. Macmillan JC, Hudson JW, Bull S, Dennis JW and Swallow CJ: Comparative expression of the mitotic regulators SAK and PLK in colorectal cancer. Ann Surg Oncol 8: 729-740, 2001.

18. Liu L,Zhang CZ,Cai M,Fu J, Chen GG and Yun J: Downregulation of polo-like kinase 4 in hepatocellular carcinoma associates with poor prognosis. PLoS One 7: e41293, 2012.

19. Zhou H, Kuang J, Zhong L, Kuo WL, Gray JW, Sahin A, Brinkley BR and Sen S: Tumour amplified kinase STK15/BTAK induces centrosome amplification, aneuploidy and transformation. Nat Genet 20: 189-193, 1998.

20. Li J, Xuan JW, Khatamianfar V, Valiyeva F, Moussa M, Sadek A, Yang BB, Dong BJ, Huang YR and Gao WQ: SKA1 over-expression promotes centriole over-duplication, centrosome amplification and prostate tumourigenesis. J Pathol 234: 178-189, 2014.

21. Shinmura K, Goto M, Suzuki M, Tao H, Yamada H, Igarashi H, Matsuura S, Maeda M, Konno H, Matsuda T, et al: Reduced expression of MUTYH with suppressive activity against mutations caused by 8-hydroxyguanine is a novel predictor of a poor prognosis in human gastric cancer. J Pathol 225: 414-423, 2011.

22. Shinmura K, Iwaizumi M, Igarashi H, Nagura K, Yamada H, Suzuki M, Fukasawa K and Sugimura H: Induction of centrosome amplification and chromosome instability in p53-deficient lung cancer cells exposed to benzo[a]pyrene diol epoxide (B[a]PDE). J Pathol 216: 365-374, 2008.

23. Li B and Dewey CN: RSEM: Accurate transcript quantification from RNA-Seq data with or without a reference genome. BMC Bioinformatics 12: 323, 2011

24. Smith JJ, Deane NG, Wu F, Merchant NB, Zhang B, Jiang A, Lu P, Johnson JC, Schmidt C, Bailey CE, et al: Experimentally derived metastasis gene expression profile predicts recurrence and death in patients with colon cancer. Gastroenterology 138: 958-968, 2010

25. Ding S, Wu X, Li G, Han M, Zhuang Y and Xu T: Efficient transposition of the piggyBac (PB) transposon in mammalian cells and mice. Cell 122: 473-483, 2005.

26. Joshi HC: Microtubule organizing centers and gamma-tubulin. Curr Opin Cell Biol 6: 54-62, 1994.

27. Oakley BR: gamma-Tubulin. Curr Top Dev Biol 49: 27-54, 2000.

28. Muzny DM, Bainbridge MN, Chang K, Dinh HH, Drummond JA, Fowler G, Kovar CL, Lewis LR, Morgan MB, Newsham IF, et al: Cancer Genome Atlas Network: Comprehensive molecular characterization of human colon and rectal cancer. Nature 487: 330-337, 2012.

29. Stewénius Y, Gorunova L, Jonson T, Larsson N, Höglund M, Mandahl N, Mertens F, Mitelman F and Gisselsson D: Structural and numerical chromosome changes in colon cancer develop through telomere-mediated anaphase bridges, not through mitotic multipolarity. Proc Natl Acad Sci USA 102: 5541-5546, 2005.

30. Chan JY: A clinical overview of centrosome amplification in human cancers. Int J Biol Sci 7: 1122-1144, 2011.

31. Yang SB, Zhou XB, Zhu HX, Quan LP, Bai JF, He J, Gao YN, Cheng SJ and Xu NZ: Amplification and overexpression of Aurora-A in esophageal squamous cell carcinoma. Oncol Rep 17: 1083-1088, 2007 
32. Gañán-Gómez I, Wei Y, Yang H, Boyano-Adánez MC and García-Manero G: Oncogenic functions of the transcription factor Nrf2. Free Radic Biol Med 65: 750-764, 2013

33. Tsai KW, Hu LY, Chen TW, Li SC, Ho MR, Yu SY, Tu YT, Chen WS and Lam HC: Emerging role of microRNAs in modulating endothelin-1 expression in gastric cancer. Oncol Rep 33: 485-493, 2015.

34. Thompson SL and Compton DA: Chromosome missegregation in human cells arises through specific types of kinetochoremicrotubule attachment errors. Proc Natl Acad Sci USA 108 17974-17978, 2011.

35. Nigg EA and Stearns T: The centrosome cycle: Centriole biogenesis, duplication and inherent asymmetries. Nat Cell Biol 13: 1154-1160, 2011

36. Nam HJ, Chae S, Jang SH, Cho H and Lee JH: The PI3K-Akt mediates oncogenic Met-induced centrosome amplification and chromosome instability. Carcinogenesis 31: 1531-1540, 2010.

37. Duensing S and Münger K: The human papillomavirus type 16 E6 and E7 oncoproteins independently induce numerical and structural chromosome instability. Cancer Res 62: 7075-7082, 2002.

38. Dykhuizen EC, Hargreaves DC, Miller EL, Cui K, Korshunov A, Kool M, Pfister S, Cho YJ, Zhao K and Crabtree GR: BAF complexes facilitate decatenation of DNA by topoisomerase II $\alpha$. Nature 497: 624-627, 2013
39. Burrell RA, McClelland SE, Endesfelder D, Groth P, Weller MC, Shaikh N, Domingo E, Kanu N, Dewhurst SM, Gronroos E, et al: Replication stress links structural and numerical cancer chromosomal instability. Nature 494: 492-496, 2013.

40. Gisselsson D, Pettersson L, Höglund M, Heidenblad M, Gorunova L, Wiegant J, Mertens F, Dal Cin P, Mitelman F and Mandahl N: Chromosomal breakage-fusion-bridge events cause genetic intratumor heterogeneity. Proc Natl Acad Sci USA 97: 5357-5362, 2000.

41. Godinho SA, Picone R, Burute M, Dagher R, Su Y, Leung CT, Polyak K, Brugge JS, Théry M and Pellman D: Oncogene-like induction of cellular invasion from centrosome amplification. Nature 510: 167-171, 2014

42. Liu J, Cheng X, Zhang Y, Li S, Cui H, Zhang L, Shi R, Zhao Z, He C, Wang C, et al: Phosphorylation of Mps1 by BRAFV600E prevents Mps1 degradation and contributes to chromosome instability in melanoma. Oncogene 32: 713-723, 2013.

43. Izumi H, Matsumoto Y, Ikeuchi T, Saya H, Kajii T and Matsuura S: BubR1 localizes to centrosomes and suppresses centrosome amplification via regulating Plk1 activity in interphase cells. Oncogene 28: 2806-2820, 2009. 